\title{
Research on Image Retrieval Technology Based on Fast Wavelet Transform
}

\author{
Liu Shuang, Yu Xiaoyang, Xiao Zhenyu and Yu Shuchun \\ The higher educational key laboratory for Measuring and Control Technology and \\ Instrumentations of Heilongjiang province, Harbin University of Science and \\ Technology, Harbin 150080, China \\ yscls2013@163.com
}

\begin{abstract}
In present research, the image feature based on adaptive wavelet has been widely used in the content-based image retrieval field. However, there is a common problem in these methods, which is to describe different query images with the same wavelet basis. In order to improve the adaptability of the image retrieval technology, we design different image basis for different query images, to achieve characterizing the feature-changing of different image categories with the adjustable distance measure. We also use the approximate Taylor expansion to reduce the seeking time of the characterization image and the characterization derivative image. As the experimental results have shown that, the new image retrieval technology with high adaptability can improve the retrieval performance greatly.
\end{abstract}

Keywords: image retrieval, wavelet transform, wavelet basis, adaptive

\section{Introduction}

Adaptive wavelet has been widely used in the content-based image retrieval, which has become a research spot in the computer science [1]. The content-based image retrieval uses the image feature to capture the similarity of two different images, and achieves the automatic retrieval in the reference data set according to the feature of the query image [2].

The early image retrieval system mainly established lower image feature with machine language and user perception [3]. The construction methods became richer after wavelet transform got used in the image retrieval technology [4]. Moreover, there is another advantage in wavelet transform, which is to flexibly adjust the wavelet basis characterizing the image feature, to make the applicability of the image retrieval method more flexible [5].

However, in current use of an image retrieval system, the construction method of query images is basically fixed, which can hardly be changed when the retrieval begins, so that the flexibility of wavelet transform is not maximized [6-7].Actually, a unified construction method of wavelet basis for different features of query images will reduce the performance of the image retrieval system inevitably [8].

Therefore, in order to improve the applicability of the image retrieval technology based on wavelet transform and the performance of the entire image retrieval system, the wavelet filter should be customized for each of the different query images. The research in this paper is launched in this starting point.

\section{Feature Extraction based on Given Wavelet Filter}

In order to facilitate the subsequent description, we use $f=\left(f_{i, j}\right)_{i=0, \ldots, W-1, j=0, \ldots H-1}$ to indicate an image with the resolution ratio of $W \times H$ pixels, and use 
$a=\left(a_{k, l}\right)_{m=-M, \ldots, M, n=-n, \ldots, n}$ to indicate a wavelet filter of $(2 M+1) \times(2 N+1)$, so that the following relationship for $w$ is true.

$$
\sum_{m=-M, n=-N}^{M, N} a_{m, n}=0
$$

According to the definition, the coefficient in wavelet transform of $f$ in anywhere $(i, j)$ and any analysis scale $l \in L^{*}$ can be calculated with the following formula.

$$
w_{i, j, l}=\sum_{\substack{m=-M, \cdots, M \\ n=-N, \cdots N,}} a_{m, n} f_{i-l_{m}, j-l_{n} *}
$$

Transform Formula (2) with Formula (1), then we can get the following formula.

$$
w_{i, j, l}=\sum_{\substack{m=-M, \cdots, M \\ n=-N, \cdots, N}} a_{m, n}\left(f_{i-l_{m}, j-l_{n}}-f_{i, j}\right)
$$

We can describe the distribution characteristics of the wavelet coefficient $w_{i, j, l}$ with the standard torque. In specific cases of the texture image, non-tilted mean generalized Gaussian function can be used to simulate an arbitrary scale wavelet coefficient distribution model. There are two parameters as $i_{c}$ and $\sigma$ in this generalized Gaussian function, which respective indicates the center of Guassian core and Guassian scale. Then Guassian function can be described as Formula (4):

$$
G\left(\left\|i-i_{c}\right\|\right)=e^{\frac{-\left\|i-i_{c}\right\|^{2}}{2 \sigma^{2}}}
$$

Then the standard torque $(\varphi, \theta)$ is related to $i_{c}$ and $\sigma$, and can be calculated with Formula (5)(6).

$$
\begin{gathered}
\varphi=\sigma \sqrt{\frac{\Re\left(3 / i_{c}\right)}{\mathfrak{R}\left(1 / i_{c}\right)}} \\
\theta=\frac{\mathfrak{R}\left(5 / i_{c}\right) \mathfrak{R}\left(1 / i_{c}\right)}{\mathfrak{R}\left(3 / i_{c}\right)^{2}}-3
\end{gathered}
$$

In which $\mathfrak{R}$ is a Gamma function, $\varphi, \theta$ is closely related to $i_{c}, \sigma$ respectively. According to the standard deviation $\varphi_{l, M, N}(f)$ and its peak value $\theta_{l, M, N}(f)$, we can describe the feature distribution of any wavelet coefficient. When $f 、 M 、 N$ are known, $\varphi_{l, M, N}(f)$ and $\theta_{l, M, N}(f)$ can be recorded as $\varphi_{l}$ and $\theta_{l}$ for short, the specific calculation is as follows:

$$
\begin{gathered}
\varphi_{l}=\sqrt{2 \delta_{l}} \\
\theta_{l}=\frac{\delta_{l}}{\varphi_{l}^{2}}
\end{gathered}
$$

In which, $\delta_{l}$ is a polynomial function of wavelet transform of $f$, the specific expression is as follows:

$$
\delta_{l}=\sum_{\substack{i \in I_{l} \\ j \in J_{l}}} \frac{l^{2}}{W H} w_{i, j, l}
$$

If $I_{l}$ and $J_{l}$ respectively indicates the set $\{0, l, 2 l, \ldots, W-1\}$ and $\{0, l, 2 l, \ldots, H-1\}$, then complexity of the image feature reflected in above formulas should be $O\left(W H M N / l^{2}\right)$. 


\section{Establishment of Characterization Image}

\subsection{Wavelet Filter Space}

Suppose that $A_{M, N}$ indicates all the wavelet filter space supporting $(2 M+1) \times(2 N+1)$, and $D$ indicates its dimensions. As the center coefficient of each filter $a \in A_{M, N}$ is limited according to Formula (1), $D=(2 M+1)(2 N+1)-1$.

\subsection{Characterization Image and Characterization Derivative Image}

According to the wavelet adaptive establishment purpose,we calculate the characterization $\left(\varphi_{l}, \theta_{l}\right)$ of $f$ with the given analysis scale $l \in N^{*}$ and each filter $a \in A_{M, N}$, the subsequent feature is called the characterization image of $f$,it is also used to calculate first-order derivative of the characterization. The subsequent characterization derivative is called the characterization derivative image of $f$.

As described previously,the image feature extracted by wavelet filter has the complexity of $O\left(W H M N / l^{2}\right)$, so that it spends much time. Therefore, we use the approximate characterization image and characterization derivative image.

\subsection{Approximate Characterization Image}

In this paper, we use the Taylor expansion to approximate characterization image and characterization derivative image, which is a new application. We calculate the characterization and characterization image with a finite set wavelet filter, which is called the key wavelet filter, and use approximate Taylor expansion in each of the other images. $A_{M, N}^{0} \subset A_{M, N}$ indicates a set of key wavelet filter, suppose that $a_{0} \in A_{M, N}^{0}$ is the key wavelet filter. The Taylor expansion of the function $F$ (no matter $\varphi_{l}, \theta_{l},\left(\partial \varphi_{l} / \partial a_{m, n}\right)$ or $\left.\left(\partial \varphi_{l} / \partial a_{m, n}\right)\right)$ at $a_{0}$ is shown as follows .

$$
F(a)=\sum_{i=0}^{n T} \frac{F^{(i)}\left(a_{0}\right)}{i !}\left(a-a_{0}\right)^{i}+O\left(\left\|a-a_{0}\right\|_{2}^{n T}\right)
$$

In which, $n T$ indicates the order of Taylor expansion in sequence, $\|\bullet\|_{2}$ indicates the second order.

$$
\|a\|_{2}=\sqrt{\frac{1}{(2 M+1)(2 N+1)} \sum_{\substack{m=-M, \cdots, M \\ n=-N, \cdots, N}} a_{m, n}^{2}}
$$

\subsection{Immutability of the Characterization Image and its Derivative}

If we can prove the characterization image and its derivative has immutability, the base of $A_{M, N}^{0}$ can be reduced.

Firstly, if multiply the wavelet filter $a \in A_{M, N}$ by a positive real number $\eta \in R^{+}$, according to Formula (3) and (9), we can get $\varepsilon_{l, d}(m, n)$ and multiply $\delta_{l}$ by $\eta^{d}, \forall l, m$ and $l$. Hence, if multiply $a$ by $\eta$, then multiply $\varphi_{l}$ by $\eta, \theta_{l}$ is immutable. For the derivative, if multiply $a$ by $\eta,\left(\partial \varphi_{l} / \partial a_{m, n}\right)$ is immutable and divide $\left(\partial k_{s} / \partial a_{m, n}\right)$ by $\eta$. 
This immutability analysis means that, we only need to calculate the characterization image and its derivative to the wavelet filter in the unit sphere $\|a\|_{2}=1$.

Secondly, if multiply $a$ by -1 , we can get $\varepsilon_{l, d}(m, n)$ and multiply $\delta_{l}$ by $(-1)^{d}, \forall l, m$ and $n$. Hence, if multiply $a$ by -1 , then $\varphi_{l}$ and $\theta_{l}$ is immutable, $\left(\partial \varphi_{l} / \partial a_{m, n}\right)$ and multiply $\left(\partial \theta_{l} / \partial a_{m, n}\right)$ by -1 .This immutability analysis means that, we only need to calculate the characterization image and its derivative to the wavelet filter in the unit hemisphere $\|a\|_{2}=1$.

\subsection{Building Steps of Characterization Image}

The first step, construct the characterization image, extract $A_{M, N}^{0} \subset A_{M, N}$ of the key wavelet filter. The calculation of the feature and its derivative related to the key filter has been described previously. Extract $A_{M, N}$ in the unit hemisphere $\|a\|_{2}=1$, the unrestrained coefficient of each filter $a$ is in $\{\tan (-(\pi / 4)+\mathrm{j}(\pi / 2 \mathrm{n})), \mathrm{j}=0, \ldots \mathrm{D}\}, i \in\{1,2, \ldots d-1, d+1, \ldots D\}$, after that, each filter $a$ is shared by $\|a\|_{2}$.

\section{Image Retrieval Process}

If $f_{q}$ indicates the query image, $\mathrm{R}$ indicates reference data set. In the image retrieval process, the characterization image is used to calculate the image similarity rank of $f \in R$, to get the retrieval results of $f_{1}\left(f_{q}\right), f_{2}\left(f_{q}\right), \ldots f_{K}\left(f_{q}\right)$, in which $K \in N^{*}$.

We suggest to mark each image with $S(f)=\left(\varphi_{l, M, N}(f), \theta_{l, M, N}(f)\right)$, then mark $S(I)$,the feature $\varphi_{l, M, N}(f)$ and $\theta_{l, M, N}(f)$, extract the analysis table at the analysis scale position $s$, and use filter $a^{l, M, N}$ supporting $(2 M+1) \times(2 N+1)$. Based on these obvious features, the similarity between $f_{q}$ and another image $f \in R$ is defined as follows:

$$
\operatorname{Sim}\left(f_{q}, f\right)=\frac{\sum_{l, M, N}\left[\varphi_{l, M, N}\left(f_{q}\right)+\theta_{l, M, N}\left(f_{q}\right)\right]}{\sum_{l, M, N}\left[\varphi_{l, M, N}(f)+\theta_{l, M, N}(f)\right]}
$$

Most of the current image retrieval methods evaluate the similarity between query image and reference with single similarity parameter. In this paper, we define a function with similarity shown in Formula (12), which is able to adjust the similarity measure pertinently according to the different above characterization images and characterization derivative images and the features of different query images, to improve the adaptability of image retrieval process greatly.

In this high adaptive image retrieval method, different similarity parameters are used in each query image. Definitely, similarity parameter (wavelet filter coefficient weight) can be used in different feature space.

\section{Experimental Results and Analysis}

In order to prove the validity of the image retrieval method based on fast wavelet transform in this paper, we build a small image retrieval library with the image data resource stored in the laboratory. The data platform has 2000 images with the resolution ratio $150 \times 150$ pixels, including landscape, insects, buildings, etc. 


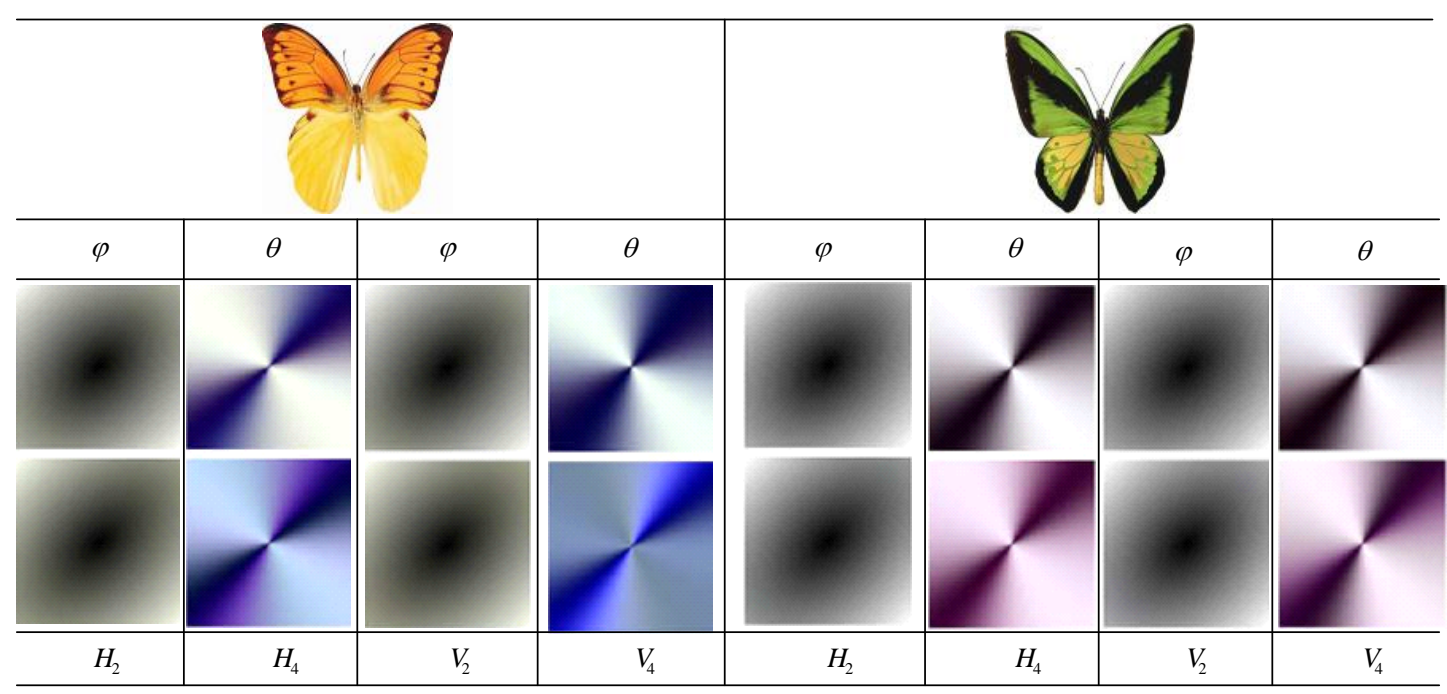

Figure 2. Extraction of Different Characterization Map

During the experiment, we use the computer with the CPU $2.0 \mathrm{GHz}$ Core Duo, RAM $8 \mathrm{G}$ and graphics with the memory 2G.Compile software platform is Visual C++.net 2005.In order to explain the realization process of the algorithm in this paper vividly, we extract the characterization images of different query images firstly, the result is shown in Figure 1.

In Figure 1, we solve the characterization image to the two different query images "butterfly" with fast wavelet transform, in which shows the characterization image of the wavelet $H_{2} 、 H_{4} 、 V_{2} 、 V_{4}$ decomposed in the scale $\varphi 、 \theta$.It can be seen in the figure that characterization images for different query images are not the same, the retrieval performance will get reduced if queried and retrieved with the same wavelet basis characterization. However, the method in this paper can design different characterization images for different query images, so that the adaptability of the entire retrieval process can be improved greatly.

Continue the further image retrieval performance test experiment with the method in this paper, the retrieval result is shown in Figure 2.

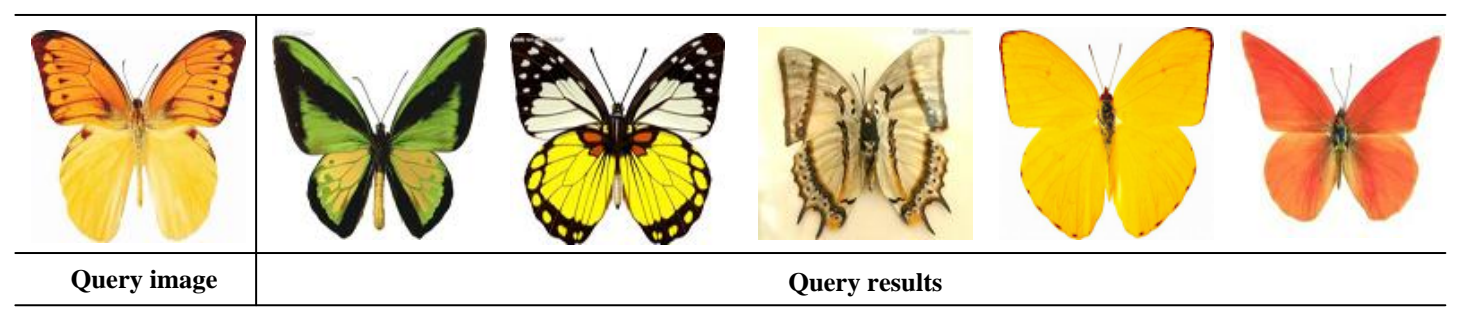

Figure 2. Query Results

Moreover, judging from the query time, querying the image "butterfly" in the image library with 2000 images only spends $0.45 \mathrm{~s}$, which does not spend extra time in calculating the characterization image.

\section{Conclusion}

It is obvious that the image retrieval performance will get reduced to use the same wavelet decomposed strategy and the unified similarity comparison for different query images. In this paper, we extract different characterization images from different query 
images and perform approximate Taylor expansion to the wavelet decomposition, to reduce time consumption in the processing procedure. As the results have shown, the image retrieval method designed in this paper has the high adaptability and improves the retrieval performance for different query images, without much extra time consumption.

\section{Acknowledgements}

This study was supported by Heilongjiang Province ordinary college training program for New Century Excellent Talents with grant number (1254-NCET-008).

\section{References}

[1] V. Chandrasekhar and G. Takacs, "CHoG: compressed histogram of gradients a low bit-rate feature descriptor [C]”, IEEE Conf. Comput. Vis. Pattern Recogn., (2009), pp. 2504-2511.

[2] M. Couze and H. Jegou, "Compact video description for copy detection with precise temporal alignment [C]", In ECCV, (2010), pp. 522-535.

[3] M. Eitz and K. Hildebrand, "Sketch-based image retrieval: benchmark and bag-of-features descriptors [J]", IEEE Trans. Vis. Comput. Graphics, vol. 17, no. 11, (2011), pp. 1624-1636.

[4] B. Girod and V. Chandrasekhar, "Mobile visual search [J]", IEEE Signal Process, Mag, vol. 28, no. 4, (2011), pp. 61-76.

[5] J. Kim and K. Grauman, "Boundary preserving dense local regions [C]", In Proc. IEEE Conf. Comput. Vis. Pattern Recogn, (2011), pp. 1553-1560.

[6] E. Rublee and V. Rabaud, "ORB: an efficient alternative to SIFT or SURF [C]", In Proc. IEEE Int. Conf. Comput. Vis., (2011), pp. 2564-2571.

[7] S. Tsai and D. Chen, "Combining image and text features: a hybrid approach to mobile book spine recognition[C]", In Proc. ACM Multimedia, (2011), pp. 1029-1032.

[8] S. Tsai and H. Chen, "Mobile visual search on printed documents using text and low bit-rate features [C]”, In Proc. IEEE Int. Conf. Image Process, (2011), pp. 2601-2604. 